\title{
Journal of Clinical Obstetrics and Gynecology Research
}

\section{Editorial}

Sonia de Miguel

\section{Institutional affiliations}

University Hospital Rio Hortega, Spain

\section{Corresponding author}

University Hospital Rio Hortega, Spain

Email: soniademiguel@yahoo.com

Received Date: Jan 17, 2020

Accepted Date: Jan 20, 2020

Published Date: Jun 13, 2020

The induction of labour, which represents an increase in maternal-fetal risks and the expense of hospital resources, has become a very frequent procedure in Obstetrics, accounting for up to $24.5 \%$ of all births in the USA in 2016.

Since EH Bishop in 1964 published her pelvic scoring in relation to the result of labor induction, there have been many authors who have studied both cervical and maternal characteristics and ultrasound measurements, with the aim of improving the predictive capacity of the Bishop score. This score is, today, the most widely used in the previous management of labour induction.

The problem with the Bishop score is that it is subjective, has a low reproducibility and produces discomfort for the patient.

Thanks to the introduction of ultrasound, we have several parameters that try to give objectivity to the different components of the Bishop score, such as the Cervical Length (CL), the Posterior Cervical Angle (PCA), Fetal-Head - Perineal Distance (FHPD), dilatation, the presence of a "funnel" image, the distance from the presentation edge to the external cervical os (EOC) and elasticity or cervical deformation (strain) obtained by a new Doppler-based tool called Doppler Imaging (TDI), entre others.

All these ultrasound variables have shown a statistically significant association with the result of labour induction, in some studies even higher than the Bishop score.

In addition to cervical characteristics, maternal and obstetric characteristics influence the success of induction and many of them have been statistically significantly related, such as maternal height, BMI, gestational age, parity, and the history of both vaginal and Caesarean section.

In an attempt to improve the prediction of labour induction, predictive mathematical models have been proposed by various authors, including multiple combinations of cervical factors (Bishop score or some component thereof), maternal (height, BMI), obstetric (parity, gestational age, previous vaginal delivery or caesarean section) and ultrasound measurements (CL, PCA, FHPD and EOC). The predictive capacity of these models is moderate-high, with areas under the curve generally between 0.61-0.82 (Pitarello et al. 71.3\%, Alvarez-Colomo et al. 82.8\%, Hiersch et al. $78.6 \%$, Levine et al. 73\%, Alavifard, in an attempt at external validation of Levine's model, $61 \%$ ).

What is really important is to be able to validate these models internally and externally, to apply them to any population, with the objective potential of not inducing patients with a very low probability of vaginal delivery.

The objective of our study was to test under routine clinical conditions the validity of a previous pilot predictive model elaborated by a single observer of our team which, based on clinical parameters (Body Mass Index, maternal height, history of previous cesarean section, previous vaginal delivery, and BS) and ultrasound parameters (CL and FHPD), resulted in a correct prediction of vaginal delivery in $82.8 \%$ of cases, with a $15 \%$ false positive rate.

A prospective, observational study was conducted between September 2010 and July 2012, recruiting 231 singleton pregnancies (Group 2), who were to initiate the IL process, according to the methodology and inclusion criteria of our previous pilot study (Group 1, 151 women). Women with a history of previous Cesarean section were included into the study. Either one out of four experienced observers performed the ultrasound examinations, and the BS was calculated by a different obstetrician or midwife who did not know the ultrasound results.

We observed that both groups were comparable according to the clinical variables analyzed and especially for those appearing in the logistic regression model of the pilot study: body mass index, maternal size, antecedent of previous $\mathrm{C}$-section, previous vaginal delivery and BS. These clinical variables were significant in relation to the outcome of IL in both groups. After applying the logistic regression parameter equation of the pilot study, the model reached a predictive capacity of $70.74 \%$, with a $20 \%$ false positive rate. These results are worse than those published in the pilot study.

In our study, the only significant differences found between groups 1 and 2 were in the measurement of ultrasound parameters. Both PCA and the existence of funnel did not show influence on the outcome of induction in Group 1, whereas FHPD showed a high significance and Odds Ratio.

Probably the discordance in FHPD is the cause of the lower predictive capacity of the model in group 2 of our study. The methodology of, especially the degree of pressure of the ultrasound probe on the soft tissues of the perineum, determines the result of the measurement, and therefore is subject to greater interobserver variability.

This possible interobserver variability of the ultrasound FHPD measurement is, admittedly, a limitation of our study, since a single observer measured FHPD in each patient.

Therefore, it has not been possible to validate internally this model in the daily conditions of clinical practice, probably due to differences in the ultrasound measurement of FHPD.

Due to these unsuccessful attempts to validate successful predictive models, they should not be used as the only tools in the assessment prior to induction of labor. 